\title{
Modelling annual maximum river flows with generalized extreme value distribution
}

\section{ABSTRACT}

A good understanding of probability distribution of annual maximum river flow is believed to improve water resources planning and design. Based on the annual maximum river flow record over 20-48 years at 9 individual river sites in Sabah, the data set are fitted into generalized extreme value (GEV) distribution with maximum likelihood estimator. Both stationary and nonstationary models are considered. Likelihood ratio test shows that most of the river flows are stationary. Over a homogeneous region, a parent distribution with common shape parameter is found well describing the behavior of selected annual maximum river flow. Hence, 10- and 100year return levels are estimated using the single model 